\title{
Diagnóstico do uso de softwares computacionais no ensino de Engenharia Química
}

\author{
M.C. Ferreira ${ }^{1}$, F. T. Vieira ${ }^{1}$ \\ 1Laboratório de Métodos Computacionais, Controle e Estimação (LAMCES), Universidade Federal do Espírito Santo, \\ Centro de Ciências Agrárias, Departamento de Engenharia Rural \\ * e-mail: thiengovieira@gmail.com
}

\begin{abstract}
Resumo
Existem inúmeros softwares disponíveis que realizam as mais diversas funções que são úteis para aplicações em problemas de Engenharia Química. A utilização dos mesmos para o ensino nas universidades torna-se cada vez mais comum com o crescente avanço tecnológico. Pelo menos uma disciplina ministrada durante o curso utiliza algum software. Para a realização desse trabalho, foi realizada uma pesquisa por meio de um questionário enviado através de correio eletrônico a professores do curso de Engenharia Química (EQ) das universidades públicas e privadas de todas as regiões do Brasil a fim de se fazer um levantamento de quais softwares são utilizados em disciplinas de EQ. Dentre todos os dados coletados, os programas mais utilizados são Excel, Matlab e $\mathrm{C} / \mathrm{C}_{++}$, sendo o Excel de uso unânime. Dessa forma, o objetivo do trabalho é realizar um diagnóstico de quais programas computacionais e qual a abrangência do uso destes nos cursos de EQ no Brasil.
\end{abstract}

\begin{abstract}
There are many softwares available that perform many different functions. These functions are useful for solve chemical engineering problems. The use of them for teaching in universities becomes increasingly common with the increasing technological advancement. At least in one subject during the course some software is used. For the realization of this article a research was conducted by sending a questionnaire to the e-mail of chemical engineering professors from public and private universities in all regions of Brazil with the purpose to make a survey of wich software are used in subjects of chemical engeneering. According to the collected data, the most usual programs are Excel, Matlab e $\mathrm{C} / \mathrm{C}++$ and Excel is used by all. Therefore, the objective is to perform a diagnostic of the computer programs and their scope in chemical engineering courses of Brazil.
\end{abstract}

Keywords: softwares, chemical engineering, universities.

\section{Introdução}

Com a revolução tecnológica a partir do final do século $\mathrm{XX}$, o mundo cada vez mais aspira conhecimento acerca das novas tecnologias que surgem a cada momento. Com isso, torna-se cada vez mais necessário que o profissional seja qualificado para desempenhar funções que dependem desse conhecimento. De acordo com Pirró e Longo [1] com as constantes mudanças tecnológicas, os indivíduos que não as acompanharem, ficarão prematuramente inabilitados para o trabalho.

Assim, é imprescindível que o método de ensino utilizado nas universidades continue a sofrer mudanças, tal como a inserção de habilidades que proporcionem ao aluno o manuseio de programas que - ajudará a resolver problemas relacionados às disciplinas por ele cursadas. Segundo Kuri [2] atualmente vivemos em um momento contraditório, em que os antigos modelos educacionais já não se sustentam e os novos ainda estão em processo de constituição e construção. A grande inovação é trazer tecnologia da informação para as universidades abandonando certos métodos obsoletos. Sobretudo na área de exatas, os alunos encontram grandes dificuldades que muitas vezes não são resolvidas com os métodos tradicionais de ensino. Nesta área, os softwares e as ferramentas computacionais de cálculos e modelagem devem ser utilizados com o objetivo de dinamizar e simplificar a matemática e muitas outras 
situações, onde se torna necessária uma maior compreensão por parte dos alunos [3].

A área de engenharia está extremamente vinculada à tecnologia. A engenharia concebe constrói e opera os meios de produção. A capacidade de "engenheirar" criações suas ou de outros primeiro, melhor e mais barato que os concorrentes é fundamental [4]. Nesse sentido, um engenheiro que faz uso de softwares na resolução dos seus problemas otimiza seu trabalho, sobretudo economizando tempo. Assim, é essencial que ao longo de todo o curso, o estudante de engenharia tenha contato com softwares que possibilitarão a realização de tarefas de maneira mais rápida e eficiente, como exige cada vez mais o mercado de trabalho.

\subsection{O uso de softwares na engenharia química}

Em um mercado competitivo e cada vez mais exigente, é necessário que o profissional de Engenharia Química recém formado esteja capacitado para enfrentar os vários desafios que o trabalho na indústria pode apresentar. Tais desafios envolvem, muitas vezes, problemas matemáticos complexos que devem ser resolvidos com a máxima eficiência e confiabilidade [5].

Para resolver os eventuais problemas, existem inúmeros softwares. Por isso, muitos professores adotam diferentes programas como uma ferramenta poderosa para lecionar as disciplinas. Um profissional de engenharia química certamente não lembrará perfeitamente como se resolve uma equação diferencial complexa que ele não se depara frequentemente, mas certamente saberá executar um programa que irá resolvê-la de maneira rápida e prática por ter convivido na graduação com o uso de softwares que executam tais tarefas. Consequentemente, esse profissional fará o mesmo para outros problemas.

\section{Metodologia}

Com o intuito de avaliar quais softwares estão sendo utilizados nos cursos de Engenharia Química do Brasil, foi enviado um formulário criado no Excel via correio eletrônico para professores e/ou coordenadores dos cursos de Engenharia Química de várias universidades públicas e privadas do país.

No formulário foi solicitado o nome da universidade, estado brasileiro em que se encontra e tipo (pública ou privada). Em seguida haviam as seguintes perguntas: Quais programas são utilizados na solução de problemas de Engenharia Química?; Em que disciplinas tais programas são utilizados?

Abaixo da primeira pergunta existiam opções de programas computacionais, assim como um espaço para que outros que não estivessem na lista fossem inseridos e abaixo da segunda questão havia nomes de disciplinas específicas de Engenharia Química e um espaço para outras não contidas na lista. Por último havia um espaço para observações e sugestões.

\section{Resultados e Discussão}

Dos questionários enviados via correio eletrônico a sessenta instituições de ensino superior do Brasil, foram obtidas dezessete respostas. Vale ressaltar que, embora tenha sido feito uma consulta extensa, o índice de retorno do questionário foi abaixo do esperado. Os nomes das instituições que enviaram suas respostas podem ser vistos na Tabela 1.

Tabela 1 - Instituições que responderam o questionário

\begin{tabular}{lll}
\hline Nome & Estado & Tipo \\
\hline UFBA & BA & Pública \\
UFAM & AM & Pública \\
UFTM & MG & Pública \\
UFU & MG & Pública \\
UFRJ & RJ & Pública \\
USP & SP & Pública \\
UFES & ES & Pública \\
UNIPAMPA & RS & Pública \\
SATC & SC & Privada \\
UCS & RS & Privada \\
UNIFRA & RS & Comunitária \\
UNIBH & MG & Privada \\
PUC & RJ & Privada \\
SENAI-CETIQT & RJ & Privada \\
UNILASALLE & RJ & Privada \\
UFTPR & PR & Pública \\
UNIPAM & MG & Privada \\
\hline
\end{tabular}

Como esperado, as respostas enviadas mostram a grande diversidade de softwares que são utilizados nas matérias específicas do curso de Engenharia Química. Isso porque existem softwares muito similares. Assim, cada professor irá optar pelo de sua preferência. Os mais adotados podem ser vistos na Figura 1. 


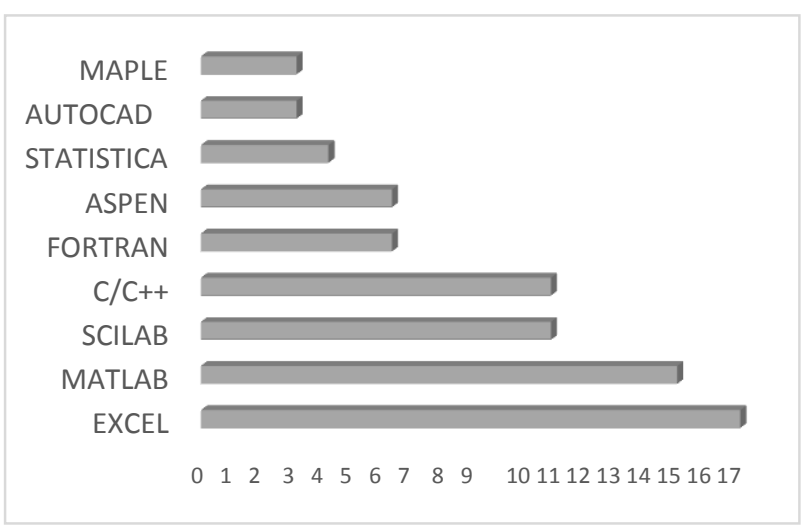

Figura 1 - Softwares utilizados pelas universidades brasileiras no ensino de Engenharia Química

Como pode ser visto, o Excel é o software mais utilizado nos cursos de Engenharia Química que participaram da pesquisa. Isso ocorre certamente pelo fato desse programa ser altamente acessível e versátil, podendo assim desempenhar inúmeras funções úteis ao estudante de Engenharia Química mesmo que em matérias do ciclo básico. Os alunos podem criar planilhas eletrônicas para executar uma série de cálculos permitindo que o trabalho seja interativo, ao contrário dos procedimentos convencionais de programação [6].

O Matlab também frequentemente usado, é um programa em que os elementos básicos de linguagem são os vetores e matrizes, além de possuir uma vasta biblioteca de funções. O Scilab, similar ao Matlab é um software livre e de código aberto para computação numérica fornecendo um poderoso ambiente de computação para aplicações de engenharia e científicas, além de incluir centenas de funções matemáticas. (SCILAB, 2015) [7]. Programas que não estão incluídos na Figura 1, como GeoGebra, Simulation CFD, DWSim, EMSO e Origin também são adotados pelas universidades.

Em relação às disciplinas da grade curricular do curso de Engenharia Química, grande parte adere a pelo menos um programa computacional. A Tabela 2 mostra alguma dessas disciplinas. O programa que cada uma utiliza dependerá do profissional que irá ministrar a disciplina. Existem programas mais simples para auxiliar o aprendizado de disciplinas do ciclo básico como Cálculo, Equações Diferenciais, Geometria Analítica e Álgebra Linear e Estatística. Pode-se usar por exemplo o Mathcad assim como ferramentas mais básicas do Excel. O importante desse resultado é que fica claro que o uso de softwares computacionais, mesmo que não sendo utilizado em todas as disciplinas do curso, vem sendo empregado nos cursos de EQ.
Tabela 2 - Disciplinas que usam softwares

\begin{tabular}{|c|}
\hline Disciplinas \\
Métodos Numéricos \\
Operações Unitárias \\
Transferência de Calor \\
Transferência de Massa \\
Cálculo de Reatores \\
Balanço de Massa e Energia \\
Controle de Processos \\
Otimização \\
Modelagem e Simulação \\
Mecânica dos Fluidos \\
Instrumentação
\end{tabular}

Embora na pesquisa disciplinas com a mesma ementa tenham sido citadas com nomes diferentes, elas foram enquadradas em algumas das citadas na Tabela 2, para facilitar a avaliação.

\section{Conclusão}

A pesquisa realizada mostra que cada instituição utiliza pelo menos um software no ensino de Engenharia Química. Embora o uso de ferramentas computacionais seja algo visto apenas nas disciplinas do ciclo professional, no curso de Engenharia Química da UFES (campus Alegre), desde o início os graduandos já tem esse contato ainda que de forma superficial e esporádica.

Fica nítido que, embora a aplicação dos softwares não seja extensiva a todas as disciplinas, o cenário atual é muito bom, tendo em vista que, se pensarmos que o resultado apresentado possa ser considerado o cenário real dos demais cursos que não participaram da pesquisa, os docentes do cursos tem incentivado o uso de softwares de alto poder computacional, mesmo sabendo que muitas vezes o processo de aprendizado e aplicação de tais programas pelos alunos seja um processo lento, seja pela própria complexidade de uso ou pela falta de aptidão no uso de recursos computacionais.

O resultado da pesquisa mostra que os cursos de EQ no Brasil tem sim buscado utilizar recursos computacionais na solução dos problemas, o que torna a formação dos alunos ainda mais completa e sintonizada com o cenário atual do mercado.

\section{Referências}

[1] LONGO, W.P. Reflexões de um engenheiro sobre ciência, tecnologia e educação. Revista do Ensino de Engenharia, v. 29, p. 40-50, 2010. 


\section{SEMANA DE ENGENHARIA QUÍMICA UFES}

[2] KURI, N.P. Tipos de personalidades e estilos de aprendizagem: proposições para 0 ensino de engenharia. 2004. 324 p. Tese (Doutorado em Engenharia de Produção) - Centro de Ciências Exatas e Tecnologia, Universidade Federal de São Carlos, São Carlos, 2004.

[3] RAMIRO, F.S.; DA COSTA, L.A.; BERNARDES, J.A. Softwares educacionais - seu uso e importância no ensino-aprendizagem dos alunos de engenharia civil. Congresso Brasileiro de Ensino de Engenharia (COBENGE), Juiz de Fora, 2014.

[4] LONGO, W.P. Educação tecnológica no mundo globalizado. Revista Engenharia, Ciência eTecnologia, ed. 14, p. 14-20, 2000.

[5] GREPINO, P.H.F.; RODRIGUES, F.A. Utilização de softwares livres no ensino de engenharia química. Revista de Engenharia Química e Química, v. 1, p. 1629, 2015.

[6] LOPES, A.; CASTRO, L.C.L.B.; NEVES, C.E.V. Utilização de planilhas eletrônicas no ensino do Método de Cross. XXXIII - Congresso Brasileiro de Ensino de Engenharia (COBENGE), Campina Grande, 2005.
[7] SCILAB. About Scilab, 2015. Disponível em: <http://www.scilab.org/scilab/about>. Acesso em: 02 fev. 2016. 